\title{
The Poincare map of randomly perturbed periodic motion
}

\author{
Pawel Hitczenko and Georgi S. Medvedev*
}

June 5, 2012

\begin{abstract}
A system of autonomous differential equations with a stable limit cycle and perturbed by small white noise is analyzed in this work. In the vicinity of the limit cycle of the unperturbed deterministic system, we define, construct, and analyze the Poincare map of the randomly perturbed periodic motion. We show that the time of the first exit from a small neighborhood of the fixed point of the map, which corresponds to the unperturbed periodic orbit, is well approximated by the geometric distribution. The parameter of the geometric distribution tends zero together with the noise intensity. Therefore, our result can be interpreted as an estimate of stability of periodic motion to random perturbations.

In addition, we show that the geometric distribution of the first exit times translates into statistical properties of solutions of important differential equation models in applications. To this end, we demonstrate three examples from mathematical neuroscience featuring complex oscillatory patterns characterized by the geometric distribution. We show that in each of these models the statistical properties of emerging oscillations are fully explained by the general properties of randomly perturbed periodic motions identified in this paper.
\end{abstract}

Keywords: Poincare map, random perturbations, limit cycle.

\section{Introduction}

Accurate description of many important dynamical phenomena in science and engineering is impossible without taking into account random factors. Even small random perturbations can transform deterministic dynamics in unexpected ways and create new asymptotic regimes, which are not present in the unperturbed deterministic system. Examples include large deviation type mechanisms of regular dynamics in randomly perturbed systems [17, 25, 39], stochastic resonance [7, 8], stochastic stabilization [24, 33], and noiseinduced synchronization [21], to name a few. Mathematical analysis of these and other related phenomena requires effective geometric theory of randomly perturbed dynamical systems, which belongs to the interface between two mathematical disciplines: the theories of dynamical systems and stochastic processes. The goal of this work is to extend the Poincare map method, the main geometric tool for studying stability of a periodic motion in deterministic systems to solutions of randomly perturbed differential equations.

\footnotetext{
${ }^{*}$ Department of Mathematics, Drexel University, 3141 Chestnut Street, Philadelphia, PA 19104, \{phitczen, medvedev\} dadrexel.edu
} 
The mathematical analysis of effects of random perturbations on dynamics of nonlinear systems was initiated by Pontryagin, Andronov, and Vitt in their pioneering paper [41]. The first systematic investigation of stability of solutions of stochastic differential equations was undertaken by Khasminsky, who extended many methods of classical theory of ordinary differential equations (cf. [31, 23]) to randomly perturbed systems [24]. Freidlin and Wentzell developed the asymptotic method of analysis of randomly perturbed dynamical systems based on large deviations estimates [18]. Asymptotic properties of solutions of stochastic ordinary differential equations were studied in [43, 19]. More recent approaches for studying randomly perturbed dynamical systems are based on ideas from the theory of dissipative dynamical systems [4] and those from the geometric theory for slow-fast systems [8]. A survey of asymptotic methods for randomly perturbed dynamical systems with a variety of applications is available in [44].

In qualitative theory of nonlinear dynamical systems, local stability analysis of invariant sets such as equilibria and periodic orbits plays an important role. There are many effective analytical techniques for studying stability of solutions of deterministic differential equations [23]. For randomly perturbed systems, stability of invariant sets is reflected in the statistics of the times of the first exit from the corresponding domains. For domains containing a stable fixed point, the Freidlin-Wentzell theory of large deviations characterizes the asymptotics of the first exit time and the geometric location of the point of exit a random trajectory from the domain [18]. Furthermore, it is known that the limiting distribution of the first exit time is exponential [13].

In the hierarchy of invariant sets of autonomous differential equations, equilibria are followed by periodic orbits. The main tool for studying stability of a periodic orbit is the Poincare map (PM). The PM captures the behavior of trajectories in a typically small neighborhood of the periodic orbit. The fixed point of the Poincare map corresponds to the periodic orbit. Stability of this fixed point of the map translates into the stability of the periodic orbit. In this work, we consider an autonomous system of differential equations in $\mathbb{R}^{n}$ possessing a limit cycle and perturbed by state-dependent white noise process. Under general assumptions on the limit cycle, we derive the PM for the randomly perturbed system and study its stability. If the periodic orbit of the deterministic system is asymptotically stable, we show that the distribution of the first exit times is approximately geometric with the parameter of the geometric distribution tending to zero together with the noise intensity. This result can be interpreted as a form of stability of motion near the periodic orbit: while the trajectories of the PM eventually leave a small neighborhood of the fixed point, for small noise they remain in this neighborhood for a very long time with large probability.

The geometric distribution of the first exit times resulting from stochastic perturbations of a stable limit cycle is important for understanding statistical properties of nonlinear oscillations generated by the randomly perturbed models. In fact, this work was motivated by our earlier analysis of a class of neuronal models in [25]. In conclusion of this paper, we discuss the applications of our results to irregular bursting and mixedmode oscillations, two important oscillatory regimes encountered in conductance based models of neurons.

The organization of the paper is as follows. Section 2 contains the formulation of the problem, preliminaries about the PM, and the statement of the main result. In Section 3, in a small neighborhood of the periodic orbit of the deterministic system, we derive a PM for the randomly perturbed problem. The analysis follows the derivation of the PM for deterministic ordinary differential equations [23] using the asymptotic expansions of solutions of stochastic differential equations on finite time intervals [9, 10, 18]. Section 4 presents the analysis of the linearization of the PM. Here, we use the results of Kesten on the iterations of linear functions of random matrices (cf. [28]) to show that the first exit time of the linearized problem has 
asymptotically geometric distribution. The analysis of this section is a substantial generalization of our previous work [25]. In Section 5, we estimate the contribution of the nonlinear terms of the PM on the statistics of the first exit times. This concludes the proof our main result - Theorem 2.7. In Section6, we illustrate the analysis of the previous sections with applications to three problems in mathematical neuroscience. To this end, we use two conductance-based models of single neurons and a model of electrically coupled network of pancreatic $\beta$-cells. In the absence of noise, all these models exhibit stable periodic oscillations. Adding noise to these models results in more complex stochastic oscillatory regimes: irregular bursting and mixedmode oscillations. We show that despite different mathematical formulations of these models and different forms of resultant oscillations, emergent stochastic oscillatory patterns in these models are formed due to the random perturbations of stable limit cycle oscillations. We verify numerically that the number of spikes in one burst and the number of small oscillations between consecutive spikes in the time series corresponding to these models are distributed approximately geometrically in accord with Theorem 2.7. We conclude this paper with a brief discussion of results of this work as well as related results in the literature in Section 7 .

\section{Assumptions and results}

\subsection{The model}

Consider an ordinary differential equation for $x: \mathbb{R} \rightarrow \mathbb{R}^{d+1}$

$$
\dot{x}=f(x),
$$

where $f: \mathbb{R}^{d+1} \rightarrow \mathbb{R}^{d+1}$ is twice continuously differentiable function, and all its partial derivatives up to the second order are uniformly bounded in $\mathbb{R}^{d+1}$. Suppose

$$
x=u(t), u(t+1)=u(t), t \in \mathbb{R}
$$

is a nonconstant periodic solution of $[2.1]$ with the least period 1 and nonvanishing derivative

$$
\dot{u}(t) \neq 0, t \in \mathbb{R} .
$$

The corresponding orbit is denoted by

$$
\mathcal{O}=\left\{x=u(\theta), \theta \in \mathbb{S}^{1}:=\mathbb{R}^{1} / \mathbb{Z}\right\} .
$$

Along with 2.1) we consider a randomly perturbed system

$$
\dot{x}_{t}=f\left(x_{t}\right)+\sigma P\left(x_{t}\right) \dot{W}_{t},
$$

where $W_{t}$ is a standard Wiener process in $\mathbb{R}^{d+1}$. Matrix $P(x) \in \mathbb{R}^{(d+1) \times(d+1)}$ is nondegenerate for any $x \in \mathbb{R}^{d+1}$. The entries of $P$ are continuously differentiable functions and all their partial derivatives are uniformly bounded in $\mathbb{R}^{d+1}$. The noise intensity $\sigma \geq 0$ is considered a small parameter. Equation 2.5) is understood in the sense of Ito [40]. 


\subsection{The local coordinates}

By Theorem VI.1.1 of [23], in a small neighborhood of $\mathcal{O}$, there is an orthonormal moving coordinate frame

$$
\left\{v(\theta), z_{1}(\theta), z_{2}(\theta), \ldots, z_{n-1}(\theta)\right\}, \quad v(\theta)=\frac{\dot{u}(\theta)}{|\dot{u}(\theta)|}, \quad \theta \in \mathbb{S}^{1} .
$$

such that the first vector $v(\theta)$ points in the tangential direction to $\mathcal{O}$. Denote

$$
Z(\theta)=\operatorname{col}\left(z_{1}(\theta), \ldots, z_{n-1}(\theta)\right) \in \mathbb{R}^{(d+1) \times d},
$$

then

$$
x=u(\theta)+Z(\theta) \rho,
$$

defines a smooth invertible transformation $x \mapsto(\theta, \rho) \in \mathbb{S}^{1} \times \mathbb{R}^{d}$ in a small neighborhood of $\mathcal{O}$ (cf. Theorem VI.1.1 of [23]).

We use the moving coordinates to rewrite [2.1] in a form more amenable for analysis.

Lemma 2.1. In new coordinates (2.7), near $\mathcal{O}$ (2.5) has the following form

$$
\begin{aligned}
& \dot{\theta}_{t}=1+a\left(\theta_{t}\right)^{\top} \rho+O\left(\left|\rho_{t}\right|^{2}\right)+\sigma\left(h\left(\theta_{t}\right)^{\top}+O\left(\left|\rho_{t}\right|\right)\right) \dot{W}_{t}, \\
& \dot{\rho}_{t}=R\left(\theta_{t}\right) \rho_{t}+O\left(\left|\rho_{t}\right|^{2}\right)+\sigma\left(H\left(\theta_{t}\right)^{\top}+O\left(\left|\rho_{t}\right|\right)\right) \dot{W}_{t},
\end{aligned}
$$

where

$$
\begin{aligned}
a^{\top}(\theta) & =2 \frac{v^{\top}(\theta)}{|f(u(\theta))|}(D f(u(\theta)))^{s} Z(\theta), \\
R(\theta) & =Z^{\top}(\theta) D f(u(\theta)) Z(\theta)-Z^{\top}(\theta) Z^{\prime}(\theta), \\
h(\theta) & =\frac{1}{|f(u(\theta))|^{2}} P(u(\theta))^{\top} f(u(\theta)) \in \mathbb{R}^{n}, \\
H(\theta) & =P(u(\theta))^{\top} Z(\theta) \in \mathbb{R}^{(d+1) \times d} .
\end{aligned}
$$

where $M^{s}:=2^{-1}\left(M+M^{\top}\right)$ stands for symmetric part of matrix $M$.

Remark 2.2. By setting $\sigma=0$ on the right hand side of (2.10) and (2.11), we obtain the deterministic equation (2.1) rewritten in the moving coordinate frame near $\mathcal{O}$.

\subsection{The Poincare map}

Our next goal is to construct the PM near the periodic orbit $\mathcal{O}$. We first review properties of the PM of the deterministic system 2.1) and then turn to the PM for the randomly perturbed system 2.5.

Throughout this subsection, we will use

$$
S_{0, \tilde{\delta}}=\{x=u(0)+Z(0) \rho:|\rho| \leq \tilde{\delta}\},
$$


to denote a local section to periodic orbit $\mathcal{O}$. Here, $\tilde{\delta}>0$ is assumed to be sufficiently small.

Using the continuity of solutions of 2.1) with respect to initial data, for sufficiently small $\delta_{1}>0$, one can find $\delta_{2} \geq \delta_{1}$ such that for any initial condition $x_{0}:=\left(0, \rho_{0}\right) \in S_{0, \delta_{1}}$, the first return time

$$
T\left(x_{0}\right)=\inf \left\{t>0: x(t) \in S_{0, \delta_{2}}\right\}
$$

is finite. The PM P : $S_{0, \delta_{1}} \rightarrow S_{0, \delta_{2}}$ is defined as follows

$$
\mathbf{P}\left(\rho_{0}\right):=\rho_{T},
$$

where $\rho_{\{0, T\}}=Z(0)^{-1} x_{\{0, T\}}$.

The following properties of $\mathbf{P}$ are well-known (see, e.g., [42]):

A) $\rho=0$ is a fixed point of $\mathbf{P}$.

B) For small $|\rho|$, the Poincare map has the following form

$$
\mathbf{P}(\rho)=A \rho+O\left(|\rho|^{2}\right), A=X(1)
$$

where $X(t)$ is the principal matrix solution of the homogeneous system

$$
\rho=R(t) \rho \quad(\text { cf. } 2.9 \text { and } 2.11) .
$$

C) If the moduli all eigenvalues of $A, \mu_{1}, \mu_{2}, \ldots, \mu_{n-1}$, are less than 1 , then periodic orbit $\mathcal{O}$ is asymptotically orbitally stable.

Next, we turn to the randomly perturbed problem $(2.5)$. Let $x(t)$ and $x_{t}:=\left(\theta_{t}, \rho_{t}\right)$ denote the solutions of the deterministic and randomly perturbed problems (2.1) and (2.5) respectively starting with with initial condition $x_{0}=\left(0, \rho_{0}\right) \in S_{0, \delta_{1}}$. Using the large deviation estimates for solutions of 2.5 ) (cf. Lemma 2.1 of Chapter 4 in [18], we have

$$
\mathbb{P}_{x_{0}}\left(\sup _{t \in[0,2]}\left|x_{t}-x(t)\right| \geq \delta_{2}\right) \leq \exp \left\{-c \sigma^{-2}\right\},
$$

for certain constant $c>0$, provided $\sigma>0$ is sufficiently small. Therefore, with exponentially close to 1 probability, the trajectory of 2.5$) x_{t}$ returns to $S_{0,2 \delta_{2}}$ after the first return time

$$
\tau_{\sigma}=\inf \left\{t>0.5: \theta_{t}=1\right\} .
$$

The first return map $\bar{\rho}=\mathbf{P}(\rho)$ is defined by

$$
\mathbf{P}(\rho):=\rho_{\tau_{\sigma}} .
$$

The following lemma provides the asymptotic description of the PM.

\footnotetext{
${ }^{1}$ Lemma 2.1 in [18] is stated for a system of the form 2.5 with $P(x)=$ id. However, the argument used in the proof of this lemma applies to systems with $P(x)$ satisfying the assumptions in $\$ 2.1$ after a suitable modification of the action functional.
} 
Lemma 2.3. The first return map has the following form

$$
\bar{\rho}=A(I+\sigma B \zeta) \rho+\sigma \eta+O\left(\sigma^{2},|\rho|^{2}\right),
$$

where

$$
\begin{gathered}
A=X(1), \quad B=X^{-1}(1) \dot{X}(1), \\
\zeta:=\xi-b(1)^{\top} \eta, \xi=\int_{0}^{1} h(s)^{\top} d W_{s}, \eta=\int_{0}^{1} X(t) X^{-1}(s) H(s)^{\top} d W_{s},
\end{gathered}
$$

and

$$
b(t)^{\top}=-\int_{0}^{t} a(s)^{\top} X(s) X^{-1}(t) d s .
$$

Remark 2.4. The principal matrix solution of the system with periodic coefficients (2.18) can be written as $X(t)=Q(t) \exp \{t A\}$, where $Q(t)$ is a 1 -periodic matrix. Therefore, $B$ in 2.23$)$ can be rewritten as follows

$$
B=Q^{-1}(0) \dot{Q}(0)+Q^{-1}(0) A Q(0) .
$$

\subsection{Stability of the randomly perturbed PM}

If the moduli of all eigenvalues of $A$ (cf. (2.16), $\mu_{1}, \mu_{2}, \ldots, \mu_{d}$, are less than 1 , then the periodic orbit $\mathcal{O}$ of the deterministic system (2.1) is asymptotically orbitally stable. This means that if the initial condition $x(0)$ is chosen sufficiently close to $\mathcal{O}$, the trajectory $\{x(t), t \geq 0\}$ will remain the vicinity of $\mathcal{O}$ for all future times. Even for very small noise intensity $\sigma>0$, for any initial condition a generic trajectory of the randomly perturbed system (2.5) eventually leaves any neighborhood of $\mathcal{O}$ due to the large deviations (cf. [18]). Nonetheless on finite interval of time $t \in[0, T], x_{t}$ with high probability exhibits stable behavior, provided $\sigma>0$ is sufficiently small and $A$ is a stable matrix. Thus, we expect that the trajectory of the PM remains close to the origin for a long time. To describe stability properties of the randomly perturbed PM, for the trajectory of $\sqrt[2.22]{2}\left\{\rho_{n}\right\}$ starting in a measurable set $D \subset \mathbb{R}^{d}$ containing the origin, we define the first exit time

$$
\tau\left(\rho_{n}, D\right)=\min \left\{n: \rho_{n} \notin D\right\}
$$

In the remainder of this subsection, we formulate two theorems characterizing the distribution of the first exit time of the trajectories of the linearized PM (Theorem 2.6) and those of the full nonlinear map (Theorem 2.7). These theorems use certain auxiliary notation, which we review next.

Definition 2.5. [25] Let $Y$ be a random variable with values in the set of positive integers and let $0<p<1$. We say that $Y$ is asymptotically geometric with parameter $p$ if

$$
\lim _{n \rightarrow \infty} \frac{\mathbb{P}(Y=n)}{\mathbb{P}(Y \geq n)}=p
$$

Recall the expression of the PM in (2.22). Along with the full nonlinear PM (2.22), we also consider its linearization

$$
\varrho_{n}=A\left(I+\sigma B \zeta_{n}\right) \varrho_{n-1}+\sigma \eta_{n}
$$


where $\left\{\zeta_{n}\right\}$ and $\left\{\eta_{n}\right\}$ are Gaussian random processes defined in 2.24).

Suppose $A$ is a stable matrix with the spectral radius

$$
\rho(A)<1-\epsilon
$$

for some $0<\epsilon<1$. Then there exists matrix norm in $\mathbb{R}^{d \times d}$ such that

$$
\|A\|^{\prime} \leq 1-\epsilon
$$

(see, for example, [26]). We will refer to the norm in (2.31) as the adapted norm.

Let $|\cdot|^{\prime}$ denote a vector norm in $\mathbb{R}^{d}$ compatible with 2.31,

$$
|A x|^{\prime} \leq(1-\epsilon)|x|^{\prime} \quad \forall x \in \mathbb{R}^{d},
$$

and $\gamma_{2} \geq \gamma_{1}>0$ such that

$$
\gamma_{1}|x| \leq|x|^{\prime} \leq \gamma_{2}|x| \quad \forall x \in \mathbb{R}^{d}
$$

For fixed $h>0$ we define

$$
D_{h}=\left\{x \in \mathbb{R}^{d}:|x|^{\prime} \leq h\right\} .
$$

It is instructive first to understand the statistics of the first exit times for the linearized PM 2.29].

Theorem 2.6. Suppose (2.30) holds and $\left\{\varrho_{n}\right\}$ denotes the trajectory of (2.29) starting from initial condition $\varrho_{0}=0$. Then for certain $\sigma_{0}=O(\epsilon)$ and for all $0<\sigma<\sigma_{0}, \tau\left(\varrho_{n}, D_{h}\right)$ is an asymptotically geometric $R V$ with parameter

$$
C_{1} \sigma \exp \left\{\frac{-C_{2}}{\sigma^{2}}\right\}\left(1+O\left(\sigma^{2}\right)\right) \leq p \leq C_{3} \sigma \exp \left\{\frac{-C_{4}}{\sigma^{2}}\right\}\left(1+O\left(\sigma^{2}\right)\right)
$$

for some positive constants $C_{1,2,3,4}$ independent from $\sigma$.

We are now in a position to formulate the main result of this paper.

Theorem 2.7. Suppose (2.30) holds and $\left\{\rho_{n}\right\}$ denotes the trajectory of (2.22) starting from initial condition $\rho_{0}=0$. Then for certain $\sigma_{0}=O(\epsilon)$ and for all $0<\sigma<\sigma_{0}, R V \tau\left(\varrho_{n}, D_{h}\right)$ is subject to the following estimate: for $n \geq 1$,

$$
\frac{\mathbb{P}(\bar{\tau}=n+1)}{\mathbb{P}(\bar{\tau} \geq n+1)} \leq p, \quad \text { where } \quad 0 \leq p \leq C\left(\sigma h^{2}\right)^{2 / 3},
$$

where $C$ is a positive constant independent of $\sigma$ and $h$.

\section{The derivation of the Poincare map}

In this section, we compute the linear part of the Poincare map of the periodic orbit $\mathcal{O}$. 


\subsection{The variational equation}

The first step in the derivation of the PM is the asymptotic approximation of the solution of 3.6 and 3.7 ) subject to initial condition

$$
\theta_{0}=0 \quad \text { and } \quad\left|\rho_{0}\right|<\delta
$$

Lemma 3.1. On any finite interval of time, for sufficiently small $\sigma>0$, the solution of the initial value problem (3.6), (3.7), and (3.1) admits the following asymptotic expansion

$$
\theta_{t}=t-b(t)^{\top} X(t) \rho_{0}+\sigma\left(\zeta_{t}+O\left(\left|\rho_{0}\right|\right)\right)+O\left(\sigma^{2},\left|\rho_{0}\right|^{2}\right), \text { and } \rho_{t}=X(t) \rho_{0}+\sigma \eta_{t}+O\left(\sigma^{2},\left|\rho_{0}\right|^{2}\right),
$$

where

$$
\eta_{t}=\int_{0}^{t} X(t, s) H(s)^{\top} d W_{s}, \xi_{t}=\int_{0}^{t} h(s)^{\top} d W_{s}, \quad \text { and } \zeta_{t}=\xi_{t}-b(t)^{\top} \eta_{t}
$$

and

$$
b(\theta)^{\top}=-\int_{0}^{\theta} a^{\top}(s) X(s) X^{-1}(\theta) d s
$$

Proof: For integration of (3.6) and (3.7), it is convenient to change $\theta$ to a new variable

$$
\phi=\theta+b(\theta)^{\top} \rho,
$$

where $b(t)$ is defined in 3.4. In new coordinates 2.10 and 2.11) become

$$
\begin{aligned}
& \dot{\phi}_{t}=1+O\left(\left|\rho_{t}\right|^{2}\right)+\sigma\left(h\left(\phi_{t}\right)^{\top}+O\left(\left|\rho_{t}\right|\right)\right) \dot{W}_{t}, \\
& \dot{\rho}_{t}=R\left(\phi_{t}\right) \rho_{t}+O\left(\left|\rho_{t}\right|^{2}\right)+\sigma\left(H\left(\phi_{t}\right)^{\top}+O\left(\left|\rho_{t}\right|\right)\right) \dot{W}_{t} .
\end{aligned}
$$

The corresponding initial condition is

$$
\phi_{0}=0 \quad \text { and } \quad\left|\rho_{0}\right|<\delta \text {. }
$$

On a finite time interval $t \in[0,2]$, we expand $x_{t}=\left(\phi_{t}, \rho_{t}\right)$ in the asymptotic sum

$$
z_{t}=z_{t}^{(0)}+\sigma z_{t}^{(1)}+\mathcal{R}(t, \sigma)
$$

where $z_{t}^{(0,1)}=\left(\phi_{t}^{(0,1)}, \rho_{t}^{(0,1)}\right)$. Function $z_{t}^{(0)}$ is the deterministic. The first order correction $z_{t}^{(1)}$ is a Gaussian process. Below we state the corresponding initial value problems for $z_{t}^{(0,1)}$. The remainder satisfies the following estimate (cf. Theorem 2.2, [18])

$$
\mathbb{E}\left\{\sup _{t \in[0,2]}|\mathcal{R}(t, \sigma)|^{2}\right\} \leq C \sigma^{2}
$$

for some positive constant $C$. We denote the asymptotic relation in 3.10 by $\mathcal{R}(t, \sigma)=O\left(\sigma^{2}\right)$.

The zeroth order problem is given by

$$
\dot{\phi}_{t}^{(0)}=1+O\left(|\rho|^{2}\right) \quad \text { and } \quad \dot{\rho}_{t}^{(0)}=R(\phi) \rho_{t}^{(0)}+O\left(\left|\rho_{t}\right|^{2}\right),
$$


subject to $x_{t}^{(0)}=\left(0, \rho_{0}\right)^{\top}$. By integrating 3.11, we have

$$
\phi_{t}^{(0)}=t+O\left(\left|\rho_{0}\right|^{2}\right), \quad \text { and } \quad \rho_{t}^{(0)}=X(t) \rho_{0}+O\left(\left|\rho_{0}\right|^{2}\right) .
$$

The first order problem has the following form

$$
\dot{\phi}_{t}^{(1)}=h(t)^{\top} \dot{W}_{t} \quad \text { and } \quad \dot{\rho}_{t}^{(1)}=R(\phi) \rho_{t}^{(1)}+H(t)^{\top} \dot{W}_{t},
$$

with initial condition $x_{t}^{(1)}=(0,0)^{\top}$. From 3.13 , we find

$$
\xi_{t}:=\phi_{t}^{(1)}=\int_{0}^{t} h(s)^{\top} d W_{s} \quad \text { and } \quad \eta_{t}:=\rho_{t}^{(1)}=\int_{0}^{t} X(t, s) H(s)^{\top} d W_{s} .
$$

Combining (3.9), 3.12) and 3.14) and switching back to the original variables $\left(\theta_{t}, \rho_{t}\right)$ (cf. (3.5)), we obtain (3.2).

\subsection{Proof of Lemma 2.3}

First, we estimate the time of the first return.

Lemma 3.2. The first return time is given by

$$
\tau_{\sigma}=1+b(1)^{\top} X(1) \rho_{0}-\sigma \zeta_{1}+o(\sigma)+O\left(\left|\rho_{0}\right|^{2}\right),
$$

Proof: Using the definition of the first return time 2.20) and the asymptotic expansion of $\theta_{t}$ (3.2), we have

$$
\tau_{\sigma}-a_{1}\left(\tau_{\sigma}\right) \rho_{0}+\zeta_{\tau_{\sigma}}+O(2)=1, \quad \text { a.s., }
$$

where $a_{1}(t):=b(t)^{\top} X(t)$ and $O(2):=O\left(\sigma^{2},\left|\rho_{0}\right|^{2}\right)$. It follows from 3.2$)$ that $\tau_{\sigma} \rightarrow \tau_{0}$ a.s., as $\sigma \rightarrow 0$, where $\tau_{0}$ is the first return time for the unperturbed deterministic trajectory $(\sigma=0)$. Thus, we write

$$
\tau_{\sigma}=\tau_{0}+\tau_{1}(\sigma)
$$

where $\tau_{1}(\sigma)=o(1)$. By plugging 3.17) in 3.15, we have

$$
\tau_{0}+\tau_{1}(\sigma)-a_{1}\left(\tau_{0}\right) \rho_{0}-a_{1}^{\prime}\left(t^{\prime}\right) \tau_{1}(\sigma) \rho_{0}+\sigma \zeta_{\tau_{0}}+\sigma\left(\zeta_{\tau_{\sigma}}-\zeta_{\tau_{0}}\right)+O(2)=1,
$$

where $t^{\prime}$ is a real number lying between $\tau_{0}$ and $\tau_{\sigma}$. By setting $\sigma=0$ in (3.18), we obtain

$$
\tau_{0}-a_{1}\left(\tau_{0}\right) \rho_{0}+O\left(\left|\rho_{0}\right|^{2}\right)=1,
$$

and, thus,

$$
\tau_{0}=1+a_{1}(1) \rho_{0}+O\left(\left|\rho_{0}\right|^{2}\right)
$$

Next,

$$
\tau_{1}(\sigma)\left(1-a_{1}\left(t^{\prime}\right) \rho_{0}\right)+\sigma \zeta_{1}+\sigma\left\{\left(\zeta_{\tau_{\sigma}}-\zeta_{\tau_{0}}\right)+\left(\zeta_{\tau_{0}}-\zeta_{1}\right)\right\}=0
$$


By the Itô isometry, two terms in the curly brackets are $o(1)$ and $O\left(\left|\rho_{0}\right|\right)$ respectively. Thus,

$$
\tau_{1}(\sigma)=-\sigma \zeta_{1}+O\left(\sigma\left|\rho_{0}\right|\right)+o(\sigma)
$$

The combination of (3.17), 3.19), and 3.20) proves the lemma.

We are now in a position to compute the first return map. From (3.2) and $(3.15)$, we have

$$
\bar{\rho}=X\left(\tau_{\sigma}\right) \rho_{0}+\sigma \eta_{\tau_{\sigma}}+O(2)=X\left(1+\sigma \zeta_{1}\right) \rho_{0}+\sigma \eta_{1}+O(2) .
$$

Further,

$$
X\left(1+\sigma \zeta_{1}\right)=X(1)\left(I+\sigma X^{-1}(1) \dot{X}(1) \zeta_{1}\right)+O\left(\sigma^{2}\right)
$$

This proves 2.22.

\section{The randomly perturbed linear map}

In this section, we study stability of the linearized PM 2.29]. Theorem 2.6 will follow from the analysis of the following slightly more general class of equations

$$
y_{n}=M_{n} y_{n-1}+z_{n}, \quad n \geq 1
$$

where

$$
M_{n}=A\left(I+\sigma \xi_{n} B\right) \quad \text { and } \quad z_{n}=\delta G \eta_{n} .
$$

Here, $A, B, G \in R^{d \times d} ; \xi_{n}$ and $\eta_{n}$ are independent identically distributed (IID) copies of standard normal $\mathrm{RV} \xi$ and $\eta$ in $\mathbb{R}$ and $\mathbb{R}^{d}$ respectively. Furthermore, we assume that $A$ is a stable matrix with spectral radius subject to 2.30 and $G$ is nondegenerate. In fact, without loss of generality, we assume that $G$ is positive definite, since otherwise, one can take $G:=\left(G G^{\top}\right)^{1 / 2}$ without changing the distribution of $z_{n}$.

Theorem 4.1. Let $h>0$ be fixed. For $y_{0} \in D_{h}$, denote the first exit time of the trajectory of (4.1) from $D_{h}$ (cf. (2.34)) by $\tau:=\tau\left(y_{n}, D_{h}\right)$. Then there exist positive $\delta_{0}=O(\epsilon)$ and $\sigma_{0}=O(\epsilon)$ such that for any $y_{0} \in D_{h}, \sigma \in\left(0, \sigma_{0}\right)$, and $\delta \in\left(0, \delta_{0}\right)$, the first exit time $\tau:=\tau\left(y_{n}, D_{h}\right)$ is asymptotically geometric $R V$ with parameter $p$ satisfying

$$
C_{1} \sigma \exp \left\{\frac{-C_{2}}{\sigma^{2}}\right\}\left(1+O\left(\sigma^{2}\right)\right) \leq p \leq C_{3} \sigma \exp \left\{\frac{-C_{4}}{\sigma^{2}}\right\}\left(1+O\left(\sigma^{2}\right)\right)
$$

for some positive constants $C_{1,2,3,4}$, which do not depend on $\sigma$ and $\delta$.

Remark 4.2. Note that setting $\delta:=\sigma$ and with appropriate choice of $G$, (4.1) coincides with the expresion of the linearized PM 2.29]. Therefore, Theorem 2.6 is a particular case of Theorem 4.1

The proof of Theorem 4.1 relies on the convergence results for iterative processes due to Kesten [28]. Specifically, our assumptions on $\left\{M_{n}\right\}$ and $\left\{z_{n}\right\}$ guarantee that $\left\{y_{n}\right\}$ converge in distribution to a random vector $y \in \mathbb{R}^{d}$ as $n \rightarrow \infty$. Furthermore, $\xi$ and $z$ are independent of $y$. We verify the conditions of the Kesten's theorem implying the convergence of $\left\{y_{n}\right\}$ in Lemma 4.3 . 
Lemma 4.3. Under our assumptions on the coefficients in (4.1), stochastic process $\left\{y_{n}\right\}$ converges in distribution to a random vector $y \in \mathbb{R}^{d}$ as $n \rightarrow \infty$, such that $(\xi, z)$ are independent of $y$.

The gist of the proof of Theorem 4.1 lies in estimating $\mathbb{P}\left(M y+z \notin D_{h}\right)$. This is the subject of the following lemma.

Lemma 4.4. There exist constants $0<C_{5}<C_{6}<1$ such that, uniformly over $s \in D_{h}$

$$
C_{5} \leq \mathbb{P}\left(M s+z \notin D_{h}\right) \leq C_{6} .
$$

We first prove Theorem 4.1 using Lemma 4.4 and then give the more technical proof of Lemma 4.4 followed by the proof of auxiliary Lemma 4.3 .

Proof: (Theorem 4.1) We need to show

$$
\lim _{n \rightarrow \infty} \frac{\mathbb{P}(\tau=n)}{\mathbb{P}(\tau \geq n)}=p>0
$$

and estimate $p$ in terms of the coefficients of Equation (4.1).

First, we note

$$
\begin{aligned}
\mathbb{P}(\tau=n+1) & =\mathbb{P}\left(y_{n+1} \notin D_{h}, y_{k} \in D_{h}, k \in[n]\right) \\
& =\mathbb{P}\left(y_{n+1} \notin D_{h} \mid y_{k} \in D_{h}, k \in[n]\right) \mathbb{P}\left(y_{k} \in D_{h}, k \in[n]\right) \\
& =\mathbb{P}\left(y_{n+1} \notin D_{h} \mid y_{k} \in D_{h}, k \in[n]\right) \mathbb{P}(\tau \geq n+1) .
\end{aligned}
$$

Further, using (4.1) and 4.2) we have

$$
\mathbb{P}\left(y_{n+1} \notin D_{h} \mid y_{k} \in D_{h}, k \in[n]\right)=\mathbb{P}\left(M_{n+1} y_{n}+z_{n} \notin D_{h} \mid y_{n} \in D_{h}\right) .
$$

The combination of (4.6) and 4.7) shows that 4.5) is equivalent to convergence of

$$
\mathbb{P}\left(M_{n+1} y_{n}+z_{n} \notin D_{h} \mid y_{n} \in D_{h}\right)=\frac{\mathbb{P}\left(M_{n+1} y_{n}+z_{n} \notin D_{h}, y_{n} \in D_{h}\right)}{\mathbb{P}\left(y_{n} \in D_{h}\right)}
$$

to a nonzero limit.

By Lemma 4.3 .

$$
\left(y_{n}, \xi_{n}, z_{n}\right) \stackrel{d}{\rightarrow}(y, \xi, z), n \rightarrow \infty,
$$

where $(\xi, z)$ is independent of $y$. Thus, the limit of the numerator in 4.8) as $n \rightarrow \infty$ is

$$
\mathbb{P}\left(M y+z \notin D_{h}, y \in D_{h}\right)=\int_{D_{h}} \mathbb{P}\left(M s+z \notin D_{h}\right) d F_{y}(s),
$$

where $F_{y}(\cdot)$ stands for the distribution function of $y$. By Lemma 4.4 .

$$
C_{5} \mathbb{P}\left(y \in D_{h}\right) \leq \int_{D_{h}} \mathbb{P}\left(M s+z \notin D_{h}\right) d F_{y}(s) \leq C_{6} \mathbb{P}\left(y \in D_{h}\right),
$$


and, therefore, the limit, $p:=\mathbb{P}\left(M y+z \notin D_{h} \mid y \in D_{h}\right)$, on the right-hand side of (4.8) satisfies

$$
0<C_{5} \leq p \leq C_{6}<1 \text {. }
$$

Proof: (Lemma 4.4)

1. Let $s \in D_{h}$, i.e., $|s|^{\prime} \leq h$. Then, using 2.32 and 4.2, we have

$$
\mathbb{P}\left(|M s+z|^{\prime} \geq h\right) \leq \mathbb{P}\left(\sigma|\xi||A B s|^{\prime}+\delta|G \eta|^{\prime}>\epsilon h\right) .
$$

Recall that $\xi \in \mathcal{N}(0,1)$ and define event

$$
\mathcal{F}=\left\{|\xi| \leq \frac{\epsilon}{2 \sigma\|A B\|^{\prime}}\right\}
$$

Using (4.11), we take the estimate in (4.10) one step further

$$
\mathbb{P}\left(|M s+z|^{\prime} \geq h\right) \leq \mathbb{P}\left(|G \eta|^{\prime}>\frac{\epsilon h}{2 \delta}, \mathcal{F}\right)+\mathbb{P}\left(\mathcal{F}^{c}\right) .
$$

We bound the second term on the right hand side of (4.12), using the normal distribution of $\xi \in$ $\mathcal{N}(0,1)$

$$
\begin{aligned}
\mathbb{P}\left(\mathcal{F}^{c}\right) & =\mathbb{P}\left(|\xi|>\frac{\epsilon}{2 \sigma\|A B\|^{\prime}}\right) \leq \mathbb{P}\left(|\xi|^{\prime}>\frac{\epsilon}{2 \sigma\|A B\|^{\prime}}\right) \\
& \leq \sqrt{\frac{2}{\pi}} \frac{\sigma}{C_{7} \epsilon} \exp \left\{\frac{-C_{7}^{2} \epsilon^{2}}{2 \sigma^{2}}\right\}\left(1+O\left(\sigma^{2} \epsilon^{-1}\right)\right),
\end{aligned}
$$

where $C_{7}=\left(2\|A B\|^{\prime}\right)^{-1}$ is independent from $\sigma$.

2. Our next goal is to bound the first term on the right hand side of (4.12). Gaussian random vector

$$
\tilde{z}=G \eta
$$

has zero mean and covariance matrix $G G^{\top}$. Denote the median of the distribution of $\tilde{z}$ by $m$ and

$$
K=\left\{x \in \mathbb{R}^{d}:|G x| \leq m\right\} \quad \text { and } \quad K_{r}=\left\{x \in \mathbb{R}^{d}: \operatorname{dist}(x, K) \leq r\right\} .
$$

We will need the following concentration inequality for Gaussian random vectors (cf. [30, pp. 19-21])

$$
\mathbb{P}\left(z \in K_{r}^{c}\right) \leq 1-\Phi(r)=\frac{\exp \left\{-2^{-1} r^{2}\right\}}{\sqrt{2 \pi} r}\left(1+O\left(r^{-2}\right)\right),
$$

where $\Phi(r)=\frac{1}{\sqrt{2 \pi}} \int_{-\infty}^{r} e^{-s^{2} / 2} d s$. We also employ an observation of Kwapien [29] to bound the median of $\tilde{z}$

$$
m^{2} \leq \mathbb{E}|G \eta|^{2}=\mathbb{E} \mathbb{T} r\left\{(G \eta)^{\top}(G \eta)\right\}=\mathbb{T} r \mathbb{E}\left(G \eta \eta^{\top} G^{\top}\right)=\|G\|_{F}^{2},
$$


where $\|G\|_{F}=\sqrt{\mathbb{T} r\left(G G^{\top}\right)}$ is the Frobenius norm of $G$. From 4.17 and the triangle inequality, for any $y \in K_{r}$ there exists $x \in K$ such that

$$
|G y| \leq|G x|+|G(y-x)| \leq\|G\|_{F}+\|G\| r .
$$

We are now in a position to use the concentration inequality (4.16) to bound the first term on the right hand side of 4.12.

First, using 2.33 we switch to the Euclidean vector norm

$$
\mathbb{P}\left(|\tilde{z}|^{\prime}>\frac{\epsilon h}{2 \delta}\right) \leq \mathbb{P}\left(|\tilde{z}|>\frac{\epsilon h}{2 \gamma_{2} \delta}\right) .
$$

Next, we specify the upper bound on $\delta$

$$
0<\delta<\frac{\epsilon h}{4\|G\|_{F} \gamma_{2}}
$$

where $\gamma_{2}$ is given by 2.33, and choose

$$
r=\frac{\epsilon h}{4 \delta \gamma_{2}\|G\|}
$$

Then, 4.20 implies that

$$
\|G\|_{F} \leq \frac{\epsilon h}{4 \delta \gamma_{2}}
$$

and 4.21 is

$$
\frac{\epsilon h}{4 \delta \gamma_{2}}=\|G\| r
$$

Hence,

$$
\frac{\epsilon h}{2 \delta \gamma_{2}}=\frac{\epsilon h}{4 \delta \gamma_{2}}+\frac{\epsilon h}{4 \delta \gamma_{2}} \geq\|G\|_{F}+\|G\| r .
$$

Thus, using 4.16) and 4.22), we obtain that the right-hand side of 4.19) is bounded by

$$
\mathbb{P}\left(|\tilde{z}|>\frac{\epsilon h}{2 \gamma_{2} \delta}\right) \leq \mathbb{P}\left(|\tilde{z}|>\|G\|_{F}+\|G\| r\right) \leq \mathbb{P}\left(K_{r}^{c}\right) \leq \frac{\exp \left\{-2^{-1} r^{2}\right\}}{\sqrt{2 \pi} r}\left(1+O\left(r^{-2}\right)\right) .
$$

Finally, by combining (4.19) and (4.23), recalling the definition of $\tilde{z}$ in (4.14), we arrive at

$$
\mathbb{P}\left(|G \eta|^{\prime}>\frac{\epsilon h}{2 \delta}\right) \leq \frac{\exp \left\{-2^{-1} r^{2}\right\}}{\sqrt{2 \pi} r}\left(1+O\left(r^{-2}\right)\right),
$$

where $r$ is specified in 4.21.

3. Combining 4.12, 4.13), and 4.24, we have

$$
\begin{aligned}
\mathbb{P}\left(M s+z \notin D_{h}\right) & \leq \sqrt{\frac{2}{\pi}} \frac{\sigma}{C_{7} \epsilon} \exp \left\{\frac{-C_{7}^{2} \epsilon^{2}}{2 \sigma^{2}}\right\}\left(1+O\left(\sigma^{2} \epsilon^{-1}\right)\right) \\
& +\frac{\delta}{\sqrt{2 \pi} C_{8} \epsilon h} \exp \left\{-\frac{C_{8}^{2} \epsilon^{2} h^{2}}{2 \delta^{2}}\right\}\left(1+O\left(\delta^{2} \epsilon^{-2} h^{-2}\right)\right)
\end{aligned}
$$


4. To get a lower bound on $\mathbb{P}\left(M s+z \notin D_{h}\right)$, we invoke the Anderson inequality [1]

$$
\mathbb{P}\left((z-x) \in D_{h}\right) \leq \mathbb{P}\left(z \in D_{h}\right) \forall x \in \mathbb{R}^{d},
$$

which is applicable since $D_{h}$ is convex and symmetric about 0 . By 4.25,

$$
\mathbb{P}\left(M s+z \notin D_{h}\right) \geq \mathbb{P}\left(|G \eta| \geq h \delta^{-1}\right) .
$$

Further,

$$
\begin{aligned}
\mathbb{P}\left(|G \eta| \geq h \delta^{-1}\right) & \geq \mathbb{P}\left(|\eta| \geq h\left(\lambda_{1}(G) \delta\right)^{-1}\right) \geq \\
\mathbb{P}\left(|z| \geq h\left(\lambda_{1}(G) \delta\right)^{-1}\right) & =\sqrt{\frac{2}{\pi}} \frac{\delta}{C_{9} h} \exp \left\{\frac{-C_{9}^{2} h^{2}}{2 \delta^{2}}\right\}\left(1+O\left(\delta^{2} h^{-2}\right)\right) .
\end{aligned}
$$

Proof: (Lemma 4.3) Recall that according to Kesten's results (see the beginning of Section 3 or Theorem 6 in [28]) for the convergence in distribution of $\left\{y_{n}\right\}$ we need to know that

$$
\mathbb{E}|G \eta|^{\gamma}<\infty \text { for some } \gamma>0 \text {, and that } \alpha:=\lim _{n \rightarrow \infty}\left\|\prod_{k=1}^{n} A\left(I+\sigma \xi_{k} B\right)\right\|<0 \text {. }
$$

(The existence of such $\alpha$ is guaranteed by the condition $\mathbb{E} \log ^{+}\|A(I+\sigma \xi B)\|<\infty$, see [20].) The first of these conditions is obviously true and holds for every $\gamma>0$. To verify the second condition, we next show

$$
\alpha:=\lim _{n \rightarrow \infty} \frac{1}{n} \log \left\|\prod_{k=1}^{n} A\left(I+\sigma \xi_{k} B\right)\right\|<0,
$$

provided

$$
\sigma<\frac{\epsilon}{c(1-\epsilon)}
$$

for a constant $c>0$ specified below.

As we mentioned, the existence of $\alpha$ is guaranteed by the integrability of $\log \|A(I+\xi B)\|$. Furthermore, by [20, Theorems 1 and 2] it suffices to show that

$$
\lim _{n \rightarrow \infty} \frac{1}{n} \mathbb{E} \log \left\|\prod_{k=1}^{n} A\left(I+\sigma \xi_{k} B\right)\right\|<0 .
$$

Obviously, we can replace $\|\cdot\|$ by any other matrix norm since neither condition is affected by passing to an equivalent norm.

We will show that (4.30) holds for the norm $\|\cdot\|^{\prime}$. Since $x \rightarrow \log x$ is concave, by Jensen's inequality

$$
\mathbb{E} \log \left\|\prod_{k=1}^{n} A\left(I+\sigma \xi_{k} B\right)\right\|^{\prime} \leq \log \mathbb{E}\left\|\prod_{k=1}^{n} A\left(I+\sigma \xi_{k} B\right)\right\|^{\prime} .
$$


Using corresponding of the adapted norm, independence of $\xi_{k}$ 's and triangle inequality we see that

$$
\mathbb{E}\left\|\prod_{k=1}^{n} A\left(I+\sigma \xi_{k} B\right)\right\|^{\prime} \leq \prod_{k=1}^{n} \mathbb{E}\left\|A\left(I+\sigma \xi_{k} B\right)\right\|^{\prime} \leq\left(\|A\|^{\prime}(1+\sigma c)\right)^{n},
$$

where $c=\|B\|^{\prime} \mathbb{E}|\xi|=\|B\|^{\prime} \sqrt{2 / \pi}$.

If $\sigma>0$ satisfies (4.29) then (2.31) implies that $\|A\|^{\prime}(1+\sigma c)<1$ for some $c>0$. Thus, by (4.30) the 4.28) holds with $\alpha \leq \log \left(\left(1-\epsilon_{1}\right)((1+\sigma c))<0\right.$.

\section{The nonlinear estimates}

In this section, we prove Theorem 2.7. To this end, we recall certain notation used in the previous sections. The full and linearized PMs (cf. (2.22) and (2.29), respectively) are given by the following difference equations:

$$
\begin{aligned}
\rho_{n} & =A\left(I+\sigma B \zeta_{n}\right) \rho_{n-1}+\sigma \eta_{n}+r\left(\rho_{n-1}\right), \\
\varrho_{n} & =A\left(I+\sigma B \zeta_{n}\right) \varrho_{n-1}+\sigma \eta_{n}
\end{aligned}
$$

where $r(\rho)=O\left(|\rho|^{2}, \sigma^{2}\right)$.

For fixed $h>0$, we define

$$
D_{h}=\left\{x \in \mathbb{R}^{d}:|x|^{\prime} \leq h\right\} .
$$

and the fist exit time $\bar{\tau}:=\tau\left(\rho_{n}, D_{h}\right)$.

Our goal is to prove the statement of the Theorem 2.7 for $n \geq 1$,

$$
\frac{\mathbb{P}(\bar{\tau}=n+1)}{\mathbb{P}(\bar{\tau} \geq n+1)} \leq p, \quad \text { where } \quad 0 \leq p \leq C\left(\sigma h^{2}\right)^{2 / 3} .
$$

As in the proof of Theorem 4.1, we obtain

$$
\mathbb{P}(\bar{\tau}=n+1)=\frac{\mathbb{P}\left(\left|\bar{\rho}_{n+1}\right|^{\prime}>h,\left|\bar{y}_{n}\right|^{\prime} \leq h\right)}{\mathbb{P}\left(\left|\bar{\rho}_{n}\right|^{\prime} \leq h\right)} \mathbb{P}(\bar{\tau} \geq n+1) .
$$

Denote $x_{n}=\rho_{n}-\varrho_{n}$ and consider the difference equation

$$
x_{n}=A\left(I+\sigma \xi_{n} B\right) x_{n-1}+r\left(\rho_{n-1}\right) .
$$

Iterating $[5.5)$, we obtain

$$
x_{n}=\left(\prod_{k=0}^{n-1} A\left(I+\sigma \xi_{n-k} B\right)\right) x_{0}+\sum_{j=2}^{n+1}\left(\prod_{k=0}^{n-j} A\left(I+\sigma \xi_{n-k} B\right)\right) r\left(\rho_{j-2}\right),
$$


where we used the convention on the right-hand side that the product over the empty range is 1 . Note that $n \leq \bar{\tau}$ implies that for $j=2, \ldots, n+1$ we have $\left|\rho_{j-2}\right|^{\prime} \leq h$. Hence, there exists a constant $C$ such that for all such $j$ 's $\left|r\left(\bar{y}_{j-2}\right)\right|^{\prime} \leq C h^{2}$. Also, since $x_{0}=0$ the first term in (5.6) vanishes. Furthermore, for $t \in \mathbb{R}$ and $w \in \mathbb{R}^{d}$

$$
|A(I+t B) w|^{\prime} \leq|A w|^{\prime}+|A(t B w)|^{\prime} \leq(1-\epsilon)\left(|w|^{\prime}+|t B w|^{\prime}\right) \leq(1-\epsilon)\left(1+|t| \cdot\|B\|^{\prime}\right)|w|^{\prime},
$$

which implies that

$$
\left|\prod_{k=0}^{n-j} A\left(I+\sigma \xi_{n-k} B\right) r\left(\rho_{j-2}\right)\right|^{\prime} \leq(1-\epsilon)^{n-j+1} \prod_{k=0}^{n-j}\left(1+\sigma\left|\xi_{n-k}\right| \cdot\|B\|^{\prime}\right)\left|r\left(\rho_{j-2}\right)\right|^{\prime} .
$$

Therefore, whenever $n \leq \bar{\tau}$, we have

$$
\left|x_{n}\right|^{\prime} \leq C h^{2} \sum_{j=2}^{n+1}(1-\epsilon)^{n-j+1} \prod_{k=0}^{n-j}\left(1+\sigma\left|\xi_{n-k}\right| \cdot\|B\|^{\prime}\right) \leq C h^{2} \sum_{\ell=0}^{\infty}\left(1-\epsilon_{1}\right)^{\ell} \prod_{k=1}^{\ell}\left(1+\sigma\left|\xi_{k}\right| \cdot\|B\|^{\prime}\right) .
$$

where $C=C(\epsilon, \sigma, A, B)$ is an absolute constant whose value may vary from use to use. We bound the variance of the latter sum as follows:

$$
\begin{aligned}
& \operatorname{var}\left(\sum_{\ell=0}^{\infty}(1-\epsilon)^{\ell} \prod_{k=1}^{\ell}\left(1+\sigma\left|\xi_{k}\right| \cdot\|B\|^{\prime}\right)\right)=\sum_{\ell=1}^{\infty}(1-\epsilon)^{2 \ell} \operatorname{var}\left(\prod_{k=1}^{\ell}\left(1+\sigma\left|\xi_{k}\right| \cdot\|B\|^{\prime}\right)\right) \\
& +2 \sum_{\ell=1}^{\infty} \sum_{m=\ell+1}^{\infty}(1-\epsilon)^{\ell+m} \operatorname{cov}\left(\prod_{k=1}^{\ell}\left(1+\sigma\left|\xi_{k}\right| \cdot\|B\|^{\prime}\right), \prod_{k=1}^{m}\left(1+\sigma\left|\xi_{k}\right| \cdot\|B\|^{\prime}\right)\right) \\
& \leq 2 \sum_{\ell=1}^{\infty} \sum_{m=\ell}^{\infty}(1-\epsilon)^{\ell+m} \operatorname{cov}\left(\prod_{k=1}^{\ell}\left(1+\sigma\left|\xi_{k}\right| \cdot\|B\|^{\prime}\right), \prod_{k=1}^{m}\left(1+\sigma\left|\xi_{k}\right| \cdot\|B\|^{\prime}\right)\right) .
\end{aligned}
$$

By independence, for $m \geq \ell \geq 0$ we have

$$
\mathbb{E} \prod_{k=1}^{\ell}\left(1+\sigma\left|\xi_{k}\right| \cdot\|B\|^{\prime}\right) \prod_{k=1}^{m}\left(1+\sigma\left|\xi_{k}\right| \cdot\|B\|^{\prime}\right)=\left(\mathbb{E}\left(1+\sigma|\xi| \cdot\|B\|^{\prime}\right)^{2}\right)^{\ell}\left(1+\sigma \mu_{B}\right)^{m-\ell}
$$

where we have set

$$
\mu_{B}=\|B\|^{\prime} \cdot \mathbb{E}|\xi|=\|B\|^{\prime} \sqrt{2 / \pi} .
$$

Therefore, the covariance above is equal to

$$
\begin{aligned}
& \left(\mathbb{E}\left(1+\sigma|\xi| \cdot\|B\|^{\prime}\right)^{2}\right)^{\ell}\left(1+\sigma \mu_{B}\right)^{m-\ell}-\left(1+\sigma \mu_{B}\right)^{m+\ell} \\
& =\left(1+\sigma \mu_{B}\right)^{m-\ell}\left\{\left(\mathbb{E}\left(1+\sigma|\xi| \cdot\|B\|^{\prime}\right)^{2}\right)^{\ell}-\left(\left(1+\sigma \mu_{B}\right)^{2}\right)^{\ell}\right\} .
\end{aligned}
$$

The term in the curly brackets is bounded above by

$$
\left(\mathbb{E}\left(1+\sigma|\xi| \cdot\|B\|^{\prime}\right)^{2}-\left(1+\sigma \mu_{B}\right)^{2}\right) \sum_{j=0}^{\ell-1}\left(\mathbb{E}\left(1+\sigma|\xi| \cdot\|B\|^{\prime}\right)^{2}\right)^{j}\left(\left(1+\sigma \mu_{B}\right)^{2}\right)^{\ell-1-j} .
$$


The factor outside the summation is equal to $\operatorname{var}\left(\sigma\|B\|^{\prime} \cdot|\xi|\right)=O\left(\sigma^{2}\right)$. To bound the sum in 5.9 we use the inequalities

$$
\left(1+\sigma \mu_{B}\right)^{2} \leq \mathbb{E}\left(1+\sigma|\xi| \cdot\|B\|^{\prime}\right)^{2}=1+2 \sigma\|B\|^{\prime} \sqrt{2 / \pi}+\left(\sigma\|B\|^{\prime}\right)^{2} \leq\left(1+\sigma\|B\|^{\prime}\right)^{2}
$$

which imply that the sum above is bounded by

$$
\sum_{j=0}^{\ell-1}\left(1+\sigma\|B\|^{\prime}\right)^{2 j}\left(1+\sigma\|B\|^{\prime}\right)^{2(\ell-1-j)} \leq \ell\left(1+\sigma\|B\|^{\prime}\right)^{2(\ell-1)} .
$$

Combining (5.7) and (5.9) with the expression bounding the variance we get

$$
C \sigma^{2} \sum_{\ell=1}^{\infty} \ell(1-\epsilon)^{2 l}\left(1+\sigma\|B\|^{\prime}\right)^{2(\ell-1)} \sum_{m=\ell}^{\infty}\left((1-\epsilon)\left(1+\sigma \mu_{B}\right)\right)^{m-\ell} .
$$

Using (5.8) we see that if $\sigma \leq \sigma_{0}$ where, say, $\sigma_{0} \leq \epsilon / 2\|B\|^{\prime}$ then the double sum in 5.10) is bounded by a constant depending on $\epsilon$ only, and thus

$$
\operatorname{var}\left(\sum_{\ell=0}^{\infty}(1-\epsilon)^{\ell} \prod_{k=1}^{\ell}\left(1+\sigma\left|\xi_{k}\right| \cdot\|B\|^{\prime}\right)\right) \leq C \sigma^{2} .
$$

Therefore, by Chebyshev's inequality, for any $h_{1}>0$ we have

$$
\mathbb{P}\left(\left|x_{n}\right|^{\prime}>h_{1}\right) \leq \mathbb{P}\left(\sum_{j=2}^{n+1}(1-\epsilon)^{n-j+1} \prod_{k=0}^{n-j}\left(1+\sigma\left|\xi_{n-k}\right| \cdot\|B\|^{\prime}\right) \geq \frac{h_{1}}{C h^{2}}\right) \leq C \sigma^{2} \frac{h^{4}}{h_{1}^{2}} .
$$

Note that if $h_{1}=O\left(\sigma^{\alpha} h^{\beta}\right)$ for $0<\alpha<1$ and $1<\beta<3 / 2$. (The optimal values of $\alpha$ and $\beta$ will be identified below.) Then the right hand side of 5.11 is $O\left(\sigma^{2(1-\alpha)} h^{2(2-\beta)}\right)$. For such $h_{1}$, set $h_{0}=h-h_{1}$. Since $\rho_{n}=\varrho_{n}+x_{n}$ we have

$$
\begin{aligned}
\mathbb{P}\left(\left|\rho_{n}\right|^{\prime} \leq h\right) & =\mathbb{P}\left(\left|y_{n}+x_{n}\right|^{\prime} \leq h\right) \geq \mathbb{P}\left(\left|y_{n}\right|^{\prime} \leq h-h_{1},\left|x_{n}\right|^{\prime} \leq h_{1}\right) \\
& =\mathbb{P}\left(\left|y_{n}\right|^{\prime} \leq h_{0}\right)-\mathbb{P}\left(\left|y_{n}\right|^{\prime} \leq h_{0},\left|x_{n}\right|^{\prime}>h_{1}\right) \\
& \geq \mathbb{P}\left(\left|y_{n}\right|^{\prime} \leq h_{0}\right)-\mathbb{P}\left(\left|x_{n}\right|^{\prime}>h_{1}\right)
\end{aligned}
$$

This bounds from below the denominator in 5.4). To upper bound the numerator $\mathbb{P}\left(\left|\rho_{n+1}\right|^{\prime}>h,\left|\rho_{n}\right|^{\prime} \leq h\right)$ we first write

$$
\mathbb{P}\left(\left|\rho_{n+1}\right|^{\prime}>h,\left|\rho_{n}\right|^{\prime} \leq h\right) \leq \mathbb{P}\left(\left|y_{n+1}\right|^{\prime}>h-h_{1},\left|y_{n}+x_{n}\right|^{\prime} \leq h\right)+\mathbb{P}\left(\left|x_{n+1}\right|^{\prime}>h_{1}\right) .
$$

Now, using that $\left|y_{n}\right|^{\prime}-\left|x_{n}\right|^{\prime} \leq\left|y_{n}+x_{n}\right|^{\prime}$ we see that $\left|y_{n}+x_{n}\right|^{\prime} \leq h$ implies that either $\left|x_{n}\right|^{\prime}>h_{1}$ or $\left|y_{n}\right|^{\prime} \leq h+h_{1}$. Therefore,

$$
\begin{aligned}
& \mathbb{P}\left(\left|y_{n+1}\right|^{\prime}>h-h_{1},\left|y_{n}+x_{n}\right|^{\prime} \leq h\right) \leq \mathbb{P}\left(\left|y_{n+1}\right|^{\prime}>h_{0},\left|y_{n}\right|^{\prime} \leq h+h_{1}\right)+\mathbb{P}\left(\left|x_{n}\right|^{\prime}>h_{1}\right) \\
& \quad \leq \mathbb{P}\left(\left|y_{n+1}\right|^{\prime}>h_{0},\left|y_{n}\right|^{\prime} \leq h_{0}\right)+\mathbb{P}\left(h-h_{1}<\left|y_{n}\right|^{\prime} \leq h+h_{1}\right)+\mathbb{P}\left(\left|x_{n}\right|^{\prime}>h_{1}\right)
\end{aligned}
$$


Combining this with 5.12 we obtain

$\frac{\mathbb{P}\left(\left|\rho_{n+1}\right|^{\prime}>h,\left|\bar{y}_{n}\right|^{\prime} \leq h\right)}{\mathbb{P}\left(\left|\rho_{n}\right|^{\prime} \leq h\right)} \leq \frac{\mathbb{P}\left(\left|y_{n+1}\right|^{\prime}>h_{0},\left|y_{n}\right|^{\prime} \leq h_{0}\right)+\mathbb{P}\left(h-h_{1}<\left|y_{n}\right|^{\prime} \leq h+h_{1}\right)+2 \mathbb{P}\left(\left|x_{n}\right|^{\prime}>h_{1}\right)}{\mathbb{P}\left(\left|y_{n}\right|^{\prime} \leq h_{0}\right)-\mathbb{P}\left(\left|x_{n}\right|^{\prime}>h_{1}\right)}$.

Using the inequality $\frac{s}{t-u} \leq \frac{s+u}{t}$ valid for all $t>0,0 \leq s \leq t$ and $0 \leq u<t$ we can bound the last expression by

$$
\frac{\mathbb{P}\left(\left|y_{n+1}\right|^{\prime}>h_{0},\left|y_{n}\right|^{\prime} \leq h_{0}\right)+\mathbb{P}\left(h-h_{1}<\left|y_{n}\right|^{\prime} \leq h+h_{1}\right)+3 \mathbb{P}\left(\left|x_{n}\right|^{\prime}>h_{1}\right)}{\mathbb{P}\left(\left|y_{n}\right|^{\prime} \leq h_{0}\right)} .
$$

Furthermore, since $y_{n}$ is a continuous random variable, $x \rightarrow \mathbb{P}\left(\left|y_{n}\right|^{\prime} \leq x\right)$ is uniformly continuous and since $h_{1}=O\left(\sigma^{\alpha} h^{\beta}\right)$ and $\mathbb{P}\left(\left|y_{n}\right|^{\prime} \leq h_{0}\right)=O(1)$ and $\mathbb{P}\left(\left|x_{n}\right|^{\prime}>h_{1}\right)=O\left(\sigma^{2(1-\alpha)} h^{2(2-\beta)}\right)$ we can bound the last quantity by

$$
\frac{\mathbb{P}\left(\left|y_{n+1}\right|^{\prime}>h_{0},\left|y_{n}\right|^{\prime} \leq h_{0}\right)}{\mathbb{P}\left(\left|y_{n}\right|^{\prime} \leq h_{0}\right)}+O\left(\sigma^{\alpha} h^{\beta}\right)+O\left(\sigma^{2(1-\alpha)} h^{2(2-\beta)}\right)
$$

To optimize the last two terms on the right hand side of $[5.13$, we choose $\alpha=2 / 3$ and $\beta=4 / 3$, which makes them $O\left(\left(\sigma h^{2}\right)^{2 / 3}\right)$. The first term on the right-hand side od 5.13 , by Theorem 2.6 , is of much smaller order. This concludes the proof of Theorem 2.7 .

\section{Applications}

In this section, we illustrate the analysis in the previous sections with applications to three problems in mathematical neuroscience. To this end, we use two conductance-based models of single neurons and a model of electrically coupled network of pancreatic $\beta$-cells. In the absence of noise, all these models exhibit stable periodic oscillations. Adding noise to these models results in more complex stochastic oscillatory regimes: irregular bursting and mixed-mode oscillations. We show that despite different mathematical formulations of these models and different forms of resultant oscillations, emergent stochastic oscillatory patterns in these models are formed due to the random perturbations of stable limit cycle oscillations. We show numerically that the number of spikes in one burst in models in $\$ 6.1$ and 6.2 and the number of small oscillations in $\S 6.3$ are distributed approximately geometrically in accord with Theorem 2.7 .

Numerical examples in $\$ 6.1$ and $\$ 6.2$ appeared before in [25, 37] respectively. We use them here to give the reader a feeling for the range of possible applications of our analytical results. The numerical example in 6.3 is new. In somewhat different dynamical regime for a stochastically forced $2 D$ FitzHugh-Nagumo oscillator, asymptotically geometric distribution of small amplitude oscillations was shown in [9] (see also [38] for related results). Finally, we note that there is another mechanism for generating irregular bursting and mixed-mode oscillations featuring geometric distribution. It does not involve random perturbations, but is based on certain properties of chaotic attractors in closely related neuronal models (see [34, 36]).

\subsection{Bursting in a single cell model}

Our first example concerns bursting. This is a common pattern of electrical activity in neurons. Bursting is characterized by series of fast oscillations separated by periods of no activity (Fig. 1 b). Understanding 

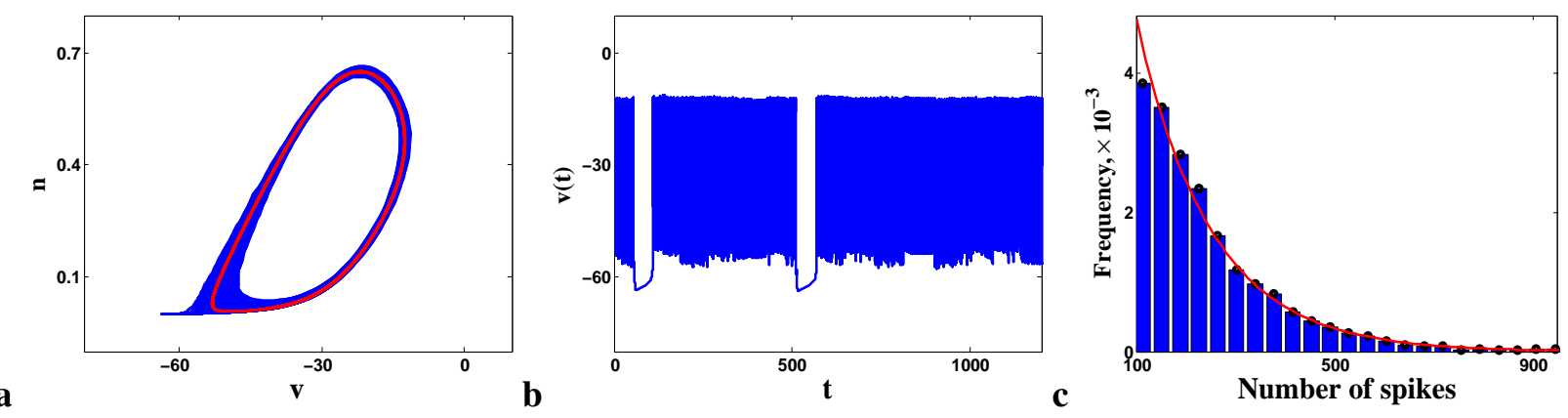

Figure 1: Randomly perturbed model of neural oscillator 6.1)-6.3. (a) The projection of a periodic trajectory of the unperturbed deterministic system (red) and that of the randomly perturbed one (blue) onto $n-v$ plane. (b) The timeseries generated by the randomly perturbed model: series of fast oscillations closely resembling oscillations of the unperturbed deterministic system are separated by periods of no activity. (c) The tail of the normalized histogram of the number of spikes in one burst is fitted with an exponential curve.

dynamical mechanisms underlying bursting is an important problem in mathematical neuroscience [27]. Below, we describe a mechanism of stochastic bursting based on random perturbations of a stable limit cycle.

The following system of three differential equations describes the dynamics of a hypothetical neuron (cf. $\S 9.1 .1,[27])$ :

$$
\begin{aligned}
C_{m} \dot{v} & =-g_{N a P} m_{\infty}(v)\left(v-E_{N a P}\right)-g_{K} n\left(v-E_{K}\right)-g_{K M} y\left(v-E_{K}\right) \\
& -g_{L}\left(v-E_{L}\right)+I+\sigma \dot{w}_{t}, \\
\dot{n} & =\frac{n_{\infty}(v)-n}{\tau_{n}} \\
\dot{y} & =\frac{y_{\infty}(v)-y}{\tau_{y}} .
\end{aligned}
$$

Here, $v, n$, and $y$ stand for the cell membrane potential and two gating variables respectively. Parameters $g_{s}$ and $E_{s}$ are the maximal conductance and the reversal potential of different ionic currents $I_{s}$, $s \in\{N a P, K, K M, L\}$ and $I$ is the applied current. The time constants $\tau_{n}$ and $\tau_{y}$ determine the rates of activation in the populations of $K$ and $K M$ channels. The steady-state functions are defined by $s_{\infty}(v)=$ $\left(1+\exp \left(\frac{a_{s}-v}{b_{s}}\right)\right)^{-1}, s \in\{m, n, y\}$. In addition, a white noise process of intensity $\sigma$ is added to the right hand side of the voltage equation to model various fluctuations affecting membrane potential.

The parameter values are summarized in the following table.

\section{Table}

\begin{tabular}{|l|c||l|c||l|c||l|l||l|c|}
\hline$g_{N a}$ & $20 m S / \mathrm{cm}^{2}$ & $g_{K}$ & $10 \mathrm{mS} / \mathrm{cm}^{2}$ & $g_{K M}$ & $5 \mathrm{mS} / \mathrm{cm}^{2}$ & $g_{L}$ & $8 \mathrm{mS} / \mathrm{cm}^{2}$ & $E_{N a}$ & $60 \mathrm{mV}$ \\
$E_{K}$ & $-90 \mathrm{mV}$ & $E_{l}$ & $-80 \mathrm{mV}$ & $\tau_{n}$ & $0.152 \mathrm{~ms}^{-1}$ & $\tau_{y}$ & $20 \mathrm{~ms}^{-1}$ & $\sigma$ & 1 \\
$\mathrm{I}$ & $5 p A$ & $a_{m}$ & $-20 \mathrm{mV}$ & $a_{n}$ & $-25 \mathrm{mV}$ & $a_{y}$ & $-10 \mathrm{mV}$ & $b_{m}$ & 15 \\
$b_{n}$ & 5 & $b_{y}$ & 5 & $C_{m}$ & $1 \mu F / \mathrm{cm}^{2}$ & & & & \\
\hline
\end{tabular}



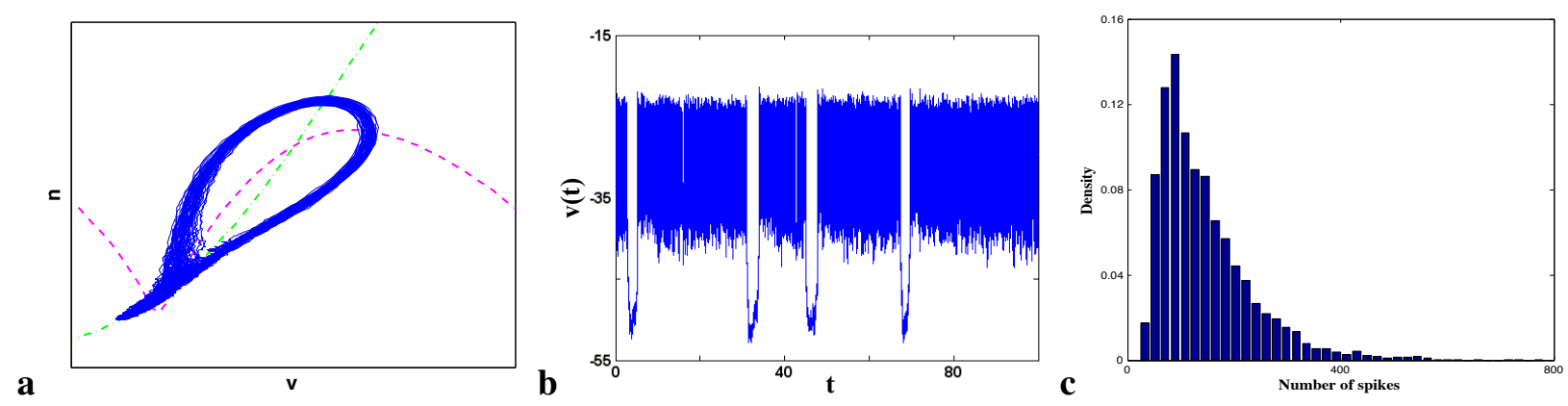

Figure 2: Randomly perturbed model of two coupled neural oscillators (6.4)-(6.6). (a) The projection of a periodic trajectory of the unperturbed deterministic system (red) and that of a randomly perturbed one (blue) onto $n-v$ plane. (b) The timeseries generated by the randomly perturbed coupled model. (c) The normalized histogram of the number of spikes in one synchronized burst features asymptotically geometric distribution.

The deterministic model (6.1)- 6.3 with $\sigma=0$ has a stable limit cycle $L$ (see Fig. 11a). Under small random perturbation $(\sigma>0)$, the trajectory of $(6.1)-(6.3)$ after a random number of rotations around the limit cycle of the deterministic system leaves the basin of attraction of the limit cycle and undergoes a large excursion in the phase space before coming back to a small neighborhood of $L$. By applying the results of this paper to the Poincare map of the limit cycle for this problem, one can show that the number of spikes in one burst is distributed approximately geometrically. The tail of the distribution of the number of spikes in one burst shown in Fig.11 is well fitted by an exponential function.

Stochastic bursting in conductance-based models similar to (6.1)- (6.3) was analyzed in [25]. The results of the paper provide a more general treatment of noise-induced bursting. Furthermore, the examples in the remainder of this section lie outside the scope of applicability of the method in [25], but can be effectively analyzed using the results of this paper.

\subsection{Bursting in a coupled network}

Our next example presents a model of a coupled network generating synchronous bursting oscillations. This is a model of an ensemble of pancreatic $\beta$ - cells coupled electrically. For simplicity, we use a twocell network and refer the interested reader to [37] for the description and numerical simulations of bigger networks.

The dynamics of Cell $i$ is governed by the following system of differential equations

$$
\begin{aligned}
C_{m} \dot{v}^{(i)} & =-I_{i o n}\left(v^{(i)}, n^{(i)}, y^{(i)}\right)+g\left(v^{(j)}-v^{(i)}\right)+\sigma \dot{w}^{(i)}, \\
\dot{n}^{(i)} & =\frac{n_{\infty}\left(v^{(i)}\right)-n^{(i)}}{\tau\left(v^{(i)}\right)}, \\
\dot{y}^{(i)} & =\epsilon\left(I_{C a}\left(v^{(i)}\right)-k y^{(i)}\right), \quad i \in\{1,2\}, j=3-i .
\end{aligned}
$$

The biophysical meaning of these equations is similar to that of the model in $6.1, v^{(i)}, n^{(i)}$, and $y^{(i)}$ stand 
for the membrane potential, gating variable, and calcium concentration corresponding to Cell $i$. Random processes $w^{(i)}, i \in\{1,2\}$, are independent copies of standard Brownian motion.

The first term on the right hand side of (6.4) models the combined effect of sodium and calcium currents, $I_{N a+C a}$, the calcium-dependent potassium current, $I_{K C a}$, delayed rectifier $I_{K}$ and a small leak current, $I_{l}$

$$
I_{i o n}=I_{N a+C a}+I_{K C a}+I_{K}+I_{l}
$$

where

$$
\begin{aligned}
I_{N a+C a} & =g_{N a+C a} m_{\infty}(v)^{3} h_{\infty}(v)\left(E_{I}-v\right), \\
I_{K C} & =\frac{g_{K C a} u}{1+u}\left(E_{K}-v\right), \\
I_{K} & =g_{K} n^{4}\left(E_{K}-v\right), \\
I_{l} & =g_{l}\left(E_{l}-v\right) .
\end{aligned}
$$

The steady state functions used to model the ionic currents above are given by

$$
f_{\infty}(v)=\frac{\alpha_{f}(v)}{\alpha_{f}(v)+\beta_{f}(v)}, \quad f \in\{m, h, n\}
$$

where

$$
\begin{gathered}
\alpha_{m}=0.1(v+25)(1-\exp \{-0.1(v+25)\})^{-1}, \beta_{m}=4 \exp \{-(v+50) / 18\}, \quad \alpha_{h}=0.07 \exp \{-0.05(v+50)\} \\
\beta_{h}=(1+\exp \{-0.1(v+20)\})^{-1}, \alpha_{n}=0.01(v+20)(1-\exp \{-0.1(v+20)\})^{-1}, \quad \beta_{n}=0.125 \exp \{-(v+30) / 80\}
\end{gathered}
$$

The time constant of the delayed rectifier is given by

$$
\tau_{n}=\left(230\left(\alpha_{n}+\beta_{n}\right)\right)^{-1} .
$$

The values of the remaining parameters are summarized in the following table.

\section{Table}

\begin{tabular}{|l|c||l|c||l|c||l|c|}
\hline$g_{N a+C a}$ & $1800 s^{-1}$ & $E_{N a+C a}$ & $100 m V$ & $g_{K}$ & $1700 s^{-1}$ & $E_{K}$ & $-75 m V$ \\
$k_{C}$ & $\frac{\{2,12\}}{18} m V$ & $g_{l}$ & $7 s^{-1}$ & $E_{l}$ & $-40 m V$ & $g_{K C}$ & $12 s^{-1}$ \\
$E_{C a}$ & $100 m V$ & $\epsilon$ & $0.03 m V^{-1} s^{-1}$ & $C_{m}$ & $1 \mu F / m^{2}$ & $\sigma$ & $10 \mathrm{mVs}^{-1}$ \\
\hline
\end{tabular}

When uncoupled $(g=0)$, the models of each cell generate stable oscillations similar to those generated by the model in 8.1 . One can show that for sufficiently strong coupling $(g \gg 1)$, the deterministic coupled system $(\sigma=0)$ has a stable limit cycle corresponding to synchronous oscillations in both cells (cf. [35]). In the presence of noise $(\sigma>0)$, the trajectory of the coupled system once in a while leaves the basin of attraction of the limit cycle. This terminates one burst. Since one spike in a burst corespond to one iteration of the PM, we conclude that the number of spikes in one synchronized burst of the coupled system must be distributed approximatelly geometrically as shown in Fig. 2 . 


\subsection{Mixed-mode oscillations}

Our final example deals with mixed-mode oscillations, another type of nonlinear oscillations that are important in neuroscience [15, 9, 36, 38]. To this end, we use a modification of the Hodgkin-Huxley model of a neuron in the regime close to the Andronov-Hopf bifurcation. It was introduced by Doi and Kumagai in [15]. The model consists of the differential equations for the membrane potential $v$ and two gating variables $n$ and $h$ :

$$
\begin{aligned}
C_{m} \dot{v} & =-g_{N a} m_{\infty}(v)^{3} h\left(v-E_{N a}\right)-g_{k} n^{4}\left(v-E_{K}\right)-g_{l}\left(v-E_{l}\right)+\sigma \dot{w} \\
\dot{n} & =\frac{n_{\infty}(v)-n}{\tau_{n}(v)} \\
\dot{h} & =\frac{h_{\infty}(v)-h}{\tau_{h}(v)} .
\end{aligned}
$$

The biophysical meaning of these equations is similar to that of the model discussed in 6.1 . The steady state functions

$$
f_{\infty}(v)=\frac{\alpha_{f}(v)}{\alpha_{f}(v)+\beta_{f}(v)}, \quad f \in\{m, h, n\}
$$

and the time constants

$$
\tau_{f}(v)=\frac{1}{\alpha_{f}(v)+\beta_{f}(v)}, \quad f \in\{h, n\}
$$

are defined using

$$
\begin{array}{rr}
\alpha_{m}=0.1(v-25)(1-\exp \{0.1(25-v)\})^{-1}, \beta_{m}=4 \exp \{-v / 18\}, & \alpha_{h}=0.07 \exp \{-0.05 v\}, \\
\beta_{h}=(1+\exp \{0.1(30-v)\})^{-1}, \alpha_{n}=0.01(v-10)(1-\exp \{0.1(10-v)\})^{-1}, & \beta_{n}=0.125 \exp \{-v / 80\} .
\end{array}
$$

The values of the remaining parameters are summarized in the following table.

\section{Table}

\begin{tabular}{|l|c||l|c||l|c||l|l||c|c|}
\hline$g_{N a}$ & $120 \mathrm{mS} / \mathrm{cm}^{2}$ & $g_{K}$ & $36 \mathrm{mS} / \mathrm{cm}^{2}$ & $g_{l}$ & $0.3 \mathrm{mS} / \mathrm{cm}^{2}$ & $E_{N a}$ & $115 \mathrm{mV}$ & $E_{K}$ & $-12 \mathrm{mV}$ \\
$E_{l}$ & $10.5999 \mathrm{mV}$ & $\bar{\tau}_{n}$ & 20 & $\bar{\tau}_{h}$ & 1 & $\sigma$ & $5 \cdot 10^{-4}$ & & \\
\hline
\end{tabular}

The parameters in the neuronal model (6.8)- 6.10$)$ are chosen such that the deterministic model $(\sigma=0)$ has a stable limit cycle, whose projection onto $v-n$ plane is shown in Fig. 33. Under the action of noise, after a random number of rotations around the periodic orbit of the deterministic system, the trajectory leaves the vicinity of the limit cycle. The global structure of the vector field of the deterministic model guarantees that the trajectory of the randomly perturbed system returns to the neighborhood of the limit cycle after each excursion in the phase space. This results in mixed-mode oscillations of the membrane potential consisting of a random number of small oscillations separated by large spikes (see Fig. 33). In accord with the results of this paper, we find that the distribution of the number of small oscillations generated by this model is approximately geometric (see Fig. 3r). 

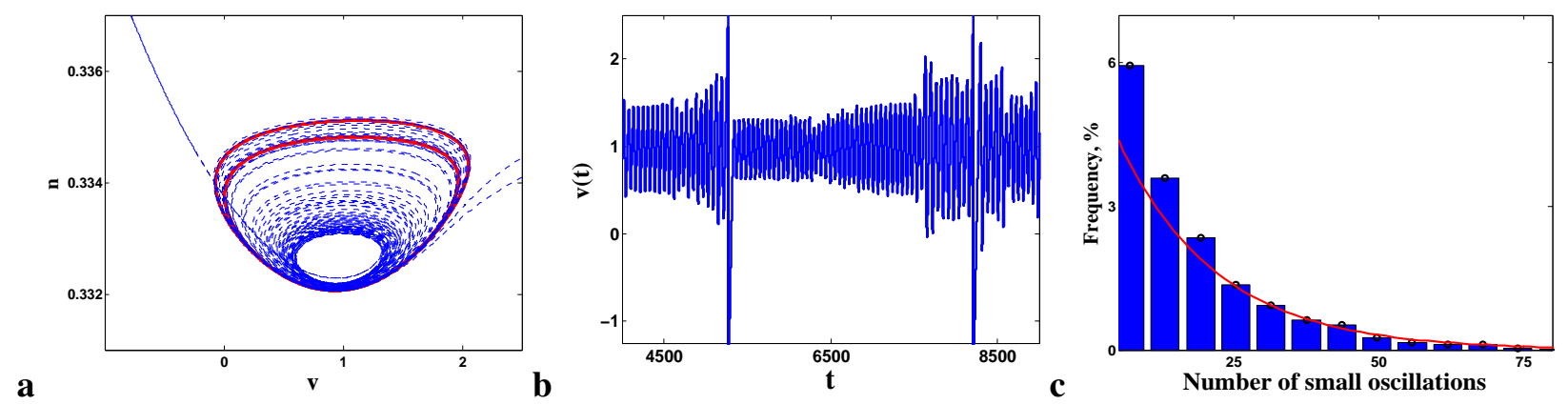

Figure 3: Noise-induced mixed-mode oscillations generated by 6.8 - 6.10$)$. (a) The projection of a periodic trajectory of the unperturbed deterministic system (red) and that of a randomly perturbed one (blue) onto $n-v$ plane. (b) The timeseries generated by the randomly perturbed coupled model. (c) The normalized histogram of the number of small oscillations between two consecutive spikes features asymptotically geometric distribution.

\section{Discussion}

Since the time of Lyapunov and Poincare, problems in nonlinear oscillations stimulated and guided the development of the geometric theory of ordinary differential equations and its diverse applications to physics and biology [2, 22, 32]. Many effective analytical methods have been developed for studying periodic motion and effects of forcing in deterministic systems [6, 12, 23]. In contrast, apart from the large deviation type techniques [18] and those for slow-fast systems [8], there are few general analytical approaches available for studying effects of random forcing on nonlinear oscillations and the analysis of such systems is often done on the case-by-case basis [5, 3, 9, 14, 25].

In the geometric theory of differential equations, the principal tool for studying stability of periodic motion is the reduction to a PM. This work presents a systematic construction of the PM for an important class of randomly perturbed problems: a limit cycle oscillator forced by small white noise. For trajectories of so-obtained PM, we analyzed the statistics of the first exit times from a small neighborhood of the origin, which corresponds to the periodic solution of the unperturbed system. We showed that if the periodic solution of the deterministic system is asymptotically stable the first exit times have approximately geometric distribution. This result implies universality of the geometric distribution in diverse oscillatory regimes generated by random perturbations of stable limit cycles.

Irregular oscillations featuring this dynamical mechanism are common for differential equation models in applied science and, in particular, in mathematical biology. We provided three representative examples from biophysics: irregular bursting and mixed-mode oscillations generated by randomly perturbed conductance-based models of neurons and synchronous noise-induced bursting in randomly forced neuronal network. We showed that these dynamical regimes are caused by random perturbations of stable limit cycles and, therefore, all of them feature geometric distribution.

Acknowledgements. This work was partially supported by a grant from the Simons Foundation (grant no. 208766 to PH) and an NSF grant (DMS 1109367 to GM). 


\section{References}

[1] T. W. Anderson, The integral of a symmetric unimodal function over a symmetric convex set and some probability inequalities. Proc. Amer. Math. Soc. 6 (1955), 170-176.

[2] A.A. Andronov, A.A. Vitt, and S.E. Khaikin, Theory of oscillations, Dover Publications, Inc., New York, 1987.

[3] J.A. Appleby, A. Rodkina, and L.-I.W. Roeger, Stability of a limit cycle for a planar system with stochastic perturbations, Func. Diff. Eqn., 16(1-2), pp. 11-28, 2009.

[4] L. Arnold, Random Dynamical Systems, Springer, Berlin, 1998.

[5] L. Arnold, P. Imkeller, and N. Sri Namachchivaya, The asymptotic stability of a noisy non-linear oscillator, J. of Sound and Vibration, 269, 1003-1029, 2004.

[6] V.I. Arnold, Geometrical methods in the theory of ordinary differential equations, second ed., Springer Verlag New York Inc., 1988.

[7] R. Benzi, A. Sutera, and A. Vulpiani, The mechanism of stochastic resonance, J. Phys. A: Math. Gen., 14:L453-L457, 1981.

[8] N. Berglund and B. Gentz, Noise-Induced Phenomena in Slow-Fast Dynamical Systems: A SamplePaths Approach, Springer, 2006.

[9] N. Berglund and D. Landon, Mixed-mode oscillations and interspike interval statistics in the stochastic FitzHugh-Nagumo model, arxiv preprint 1105.1278, 2011.

[10] Yu. N. Blagoveshchenskii, Diffusion processes depending on small parameter, Theory Probab. Appl., 7 (1962), pp. 130-146.

[11] Yu. N. Blagoveshchenskii and M. Freidlin, On certain properties of diffusion processes depending on small parameter, Soviet Mathematics, 2(3), 633-636, 1961.

[12] S.-N. Chow and J.K. Hale, Methods Of Bifurcation Theory, Springer-Verlag New York Inc, New York, 1982.

[13] M.V. Day, On the exponential law in the small parameter exit problem, Stochastics, 8 pp. 297-323, 1983.

[14] L. DeVille, N.S. Namachchivaya and Zoi Rapti, Stability of a Stochastic Two-Dimensional NonHamiltonian System, SIAM Journal of Applied Math., to appear.

[15] S. Doi and S. Kumagai, Generation of very slow neuronal rhythms and chaos near the Hopf bifurcation in single neuron models, J. of Comp. Neurosci., 19, 325-356, 2005.

[16] W. Feller, An Introduction to Probability Theory and Its Applications, vol. I. Wiley, 3rd edition, 1968.

[17] M.I. Freidlin, On stable oscillations and equilibriums induced by small noise, J. of Stat. Phys., 103 (1-2), 283-300, 2001. 
[18] M.I. Freidlin and A.D. Wentzell, Random perturbations of dynamical systems, 2nd ed., Springer, New York, 1998.

[19] A. Friedman, Stochastic Differential Equations and Applications, Dover Publications, Inc., Mineola, New York, 2006.

[20] H. Furstenberg and H. Kesten, Products of random matrices, Ann. Math. Statist., 31: 457-469, 1960.

[21] D.S. Goldobin and A. Pikovsky, Synchronization and desynchronization of self-sustained oscillators by common noise, Phys. Rev. E 71, 045201, 2005.

[22] J.K Hale, Oscillations in nonlinear systems, McGraw-Hill Book Company, Inc., 1963.

[23] J.K Hale, Ordinary Differential Equations, Krieger, 2nd edition, 1980.

[24] R.Z. Has'minskii, Stochastic stability of differential equations, Sijthoff \& Noordhoff, Rockville, MD, 1980.

[25] P. Hitczenko and G.S. Medvedev, Bursting oscillations induced by small noise, SIAM J. Appl. Math., 69(5): 1359-1392, 2009.

[26] R. A. Horn and C. A. Johnson. Matrix Analysis, Cambridge University Press, 1985.

[27] E.M. Izhikevich, Dynamical systems in neuroscience: the geometry of excitability and bursting, MIT Press, Cambridge, MA, 2007.

[28] H. Kesten, Random difference equations and renewal theory for products of random matrices, Acta Math., 131, 207-248, 1973.

[29] S. Kwapień. A remark on the median and the expectation of convex functions of Gaussian vectors, Probability in Banach Spaces IX, Birkhäuser, 1994, pp. 271-272.

[30] M. Ledoux and M. Talagrand Probability in Banach spaces, Springer, 1991.

[31] I.G. Malkin, The theory of stability of motion, second edition, Editorial, Moscow, 2004 (in Russian).

[32] I.G. Malkin, Methods of Lyapunov and Poincare in the theory of nonlinear oscillations, second ed., Editorial, Moscow, 2004 (in Russian)

[33] X. Mao, Stochastic stabilization and destabilization, Systems and Control Letters, 23, 279-290, 1994.

[34] G.S. Medvedev, Transition to bursting via deterministic chaos, Phys. Rev. Lett., 97, 048102, 2006.

[35] G.S. Medvedev, Synchronization of coupled limit cycles, J. Nonlin. Sci., 21, 3, 441-464, 2011.

[36] G.S. Medvedev and Yun Yoo, Chaos at the border of criticality, Chaos, 18, 033105, 2008.

[37] G.S. Medvedev and S. Zhuravytska, Shaping bursting by electrical coupling and noise, arXiv:1111.0642, 2011.

[38] C.B. Muratov and E. Vanden-Eijnden, Noise-induced mixed-mode oscillations in a relaxation oscillator near the onset of a limit cycle, Chaos, 18, 015111, 2008. 
[39] C.B. Muratov, E. Vanden Eijnden, and W. E, Self-induced stochastic resonance in excitable systems, Physica D 210, 227-240, 2005.

[40] B. Øksendal. Stochastic differential equations, Springer, 6th edition, 2003.

[41] L.S. Pontriagin, A.A. Andronov, and A.A. Vitt, O statitisticheskom rassmotrenii dinamicheskikh sistem, Zh. Eksper. Teoret. Fiz., 3(3), pp. 165-180 (in Russian).

[42] L.P. Shilnikov, A.L. Shilnikov, D.V. Turaev, and L.O. Chua, Methods of qualitative theory in nonlinear dynamics, Part I, World Scientific, 1998.

[43] A.V. Skorokhod, Asymptotic methods in the theory of stochastic differential equations, AMS, 1989.

[44] A.V. Skorokhod, F.C. Hoppensteadt, and Salehi, Random perturbation methods, Springer-Verlag, New York, 2002. 